\title{
Performance analysis of Strain sensor based on Fiber Bragg Grating
}

\author{
Aniket Shivram More ${ }^{l}$, Pritam sanjay Lad ${ }^{2}$, Shivram RamnarayananKrishnan ${ }^{3}$, and Savita R. Bhosale ${ }^{4 *}$ \\ ${ }^{1}$ Department of Electronics, Ramrao Adik Institute of Technology,Navi Mumbai India \\ ${ }^{2}$ Department of Electronics, Ramrao Adik Institute of Technology, Navi Mumbai India \\ ${ }^{3}$ Department of Electronics, Ramrao Adik Institute of Technology, Navi Mumbai India \\ ${ }^{4}$ Department of Electronics, Ramrao Adik Institute of Technology, Navi Mumbai India
}

\begin{abstract}
A fiber Bragg grating can be worked as reflector, it is constructed in a short section of optical fiber that reflects light of specific wavelength and allow to pass remaining wavelengths. This is possible by creating a periodic change in refractive index within the fiber core. The change in the modal index of fiber caused due to strain results in a Bragg wavelength. This work describes performance of Fiber Bragg Gratings as a strain sensor at $1550 \mathrm{~nm}$ wavelength is considered for simulation, corresponding dynamic strain and wavelength shifts are analysed. This performance analysis is useful especially for intrusion detection system where environments are harsh. This sensor is compatible with data communication system and can be used for remote sensing.
\end{abstract}

Keywords: Fiber Bragg Grating, Optical sensors, Strain, Wavelength

\section{Introduction}

The Optical fiber supported sensors gaining attention due to its advantages than existing sensing technologies with improved sensitivity. FBG sensors are not affected by electromagnetic interference, this characteristics improves accuracy of the intrusion detection system. The uses of Optical fiber sensors are receiving attention to deploy traditional electrical and mechanical sensors for monitoring parameters. In FBG based sensors the core of single mode fiber is exposed to a periodic pattern the exposure creates variation in refractive index of fibers core which results in fixed index modulation called grating. The working principle of FBG is wavelengths of the output signal shifted with change in strain [1-2].

Fibers explored many applications in remote sensing. In few applications, optical fiber itself can act as sensor.

Fiber optics based sensors are popular because of its small size, light weight and free from electromagnetic and electrical interference.

The basic principle of working of FBG sensors are change in the field of the grating or the change in the refractive index directly proportional to the change in the Bragg wavelength. Thus physical parameter change can be sensed using a FBG, by measuring the change in the Bragg wavelength or the change in reflection coefficient of a particular wavelength [3]. ER-FBGs sensor with different diameters build good strain sensor for high temperature environments strain sensor like aerospace engines, and high temperature pipelines in the oil and gas industry[4].

A FBG strain sensors network can be used for security purpose on railway track. FBG sensors do not need electrical power. These sensors have multiplexing capability. Due to this characteristic it offers cost effective solution to security systems. A high intrinsic sensitivity FBG sensor configuration offers many advantages whenever simultaneous measurements are required. FBG sensors are cost effective and easy to fabricate [5-6].

\section{Literature Survey}

An optical fiber sensor has the potential to overcome drawbacks of conventional electric sensors. A variety of of optical fiber sensors based FBG have been proposed based on incident lights modulation parameters.

A PS-FBG sensor system with high-sensitivity ultrasonic was proposed and demonstrated. The variable wavelength of the TLS were used, the reflected and transmitted optical powers of the sensor was balanced. The Fiber Bragg Gratings (FBGs) sensors have ability to look after big areas to secure from unauthorized actions in railway scenarios. This broad-bandwidth quasi-static FBG strain sensor with dual-comb spectroscopy was

Corresponding author: $\underline{\text { savita.bhosale@ rait.ac.in }}$ 
proposed. This 1-THz bandwidth sensor can be used for geophysics applications.

There are various sensing techniques explored depending on different systems such as transmitting systems, such as wired security systems and optical fiber security systems. Earlier wired security systems were considered the most reasonable and consistent systems, although they usually have to be set by expert to make sure wires, run from each sensor to the control panel,

The advantage offered by wired systems is they can be effortlessly linked to a display device using a telephone line.

A sensitivity-enhanced fiber-optic based strain sensor with an etched and regenerated FBG (ER-FBG) was shown experimentally which can be used for temperature measurements. The processes of chemical etching provide regenerated gratings.

Optical fiber based security system gives many advantages where as optical fiber can act not only transmission medium but also sensor. It is lightweight, very small size, ability to work in rough environment, immune to radio frequency interference, electromagnetic interference, requires low power, having large bandwidth, its capability to interface data communication system and much more sensitive than traditional sensors. This work focuses on how FBG can be used as strain sensor. The FBG based sensor especially more sensitive to strain variation.

A new sensing configuration was proposed for parallel measurement of strain and temperature based on a Bragg grating duo, with one grating in the etched and the other in un-etched region of polymer fibre. This sensor configuration recommends a number of advantages over earlier reported techniques, including high intrinsic sensitivity, low cost and eases in fabrication.

The FBGs are highly sensitive towards various ecological parameters, considering physical, chemical, biomedical, electrical parameter and these sensors can be used for monitoring structural health in civil infrastructure and interrogation techniques.

A high-resolution concurrent strain and temperature measurement system using $\pi$-phase-shifted fiber Bragg grating was proposed and designed.

\section{Theory and Working Principle}

The Fig. 1 shows Fiber Bragg Gratings with refractive index $\mathrm{n} 0, \mathrm{n} 1, \mathrm{n} 2$ and $\mathrm{n} 3$.Spectral response of input, transmitted signal and reflected is also illustrated.

The general equation of the FBG is

$$
\lambda B=2 \eta \operatorname{eff} \Lambda
$$

Where

$\lambda \mathrm{B}$ is Bragg wavelength

$\eta$ peff is effective refractive index of the core

$\Lambda$ is the grating period.

If Fiber Bragg condition is not satisfied, the light from each subsequent plane in the grating will be out of phase and cancel.

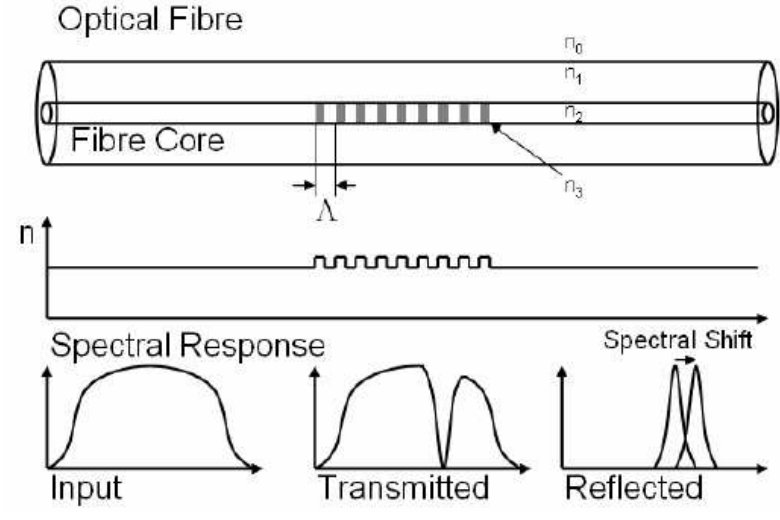

Fig. 1. Fiber Bragg Grating

The sensitivity of the Bragg wavelength strain can be calculated as below

$$
\Delta \lambda_{\mathrm{B}}=\left[\lambda \frac{\delta \mathrm{n}}{\delta \mathrm{t}}+\mathrm{n} \frac{\delta \Delta}{\delta \mathrm{x}}\right] \Delta \mathrm{l}
$$

$\Delta \mathrm{l}$ is differences of Fiber Bragg Gratings length affected by strain[7-8].

\section{Sensing Principle}

The Fiber Bragg grating is widely employed for sensing applications. The main principle used in FBG based sensor is to analyse the shift in Bragg wavelength. The Bragg wavelength of an optical fiber grating is depends on function of grating period $\Lambda$.

Thus any change in refractive index directly proportional the Fiber Bragg wavelength and this change can be obsereved in display of transmitted or reflected signals spectrum, also any change in the grating period due to external strain will change Bragg wavelength.

The basic principle of FBG is measurement of the peak wavelength shift due to applied strain. Single mode FBG at $1550 \mathrm{~nm}$ is used for analysis of strain performance. The selected wavelength of $1550 \mathrm{~nm}$ offers less attenuation and absorption losses. The proposed system is designed and its performance is analyzed by using Optisystem software [9-10].

\section{Block Diagram}

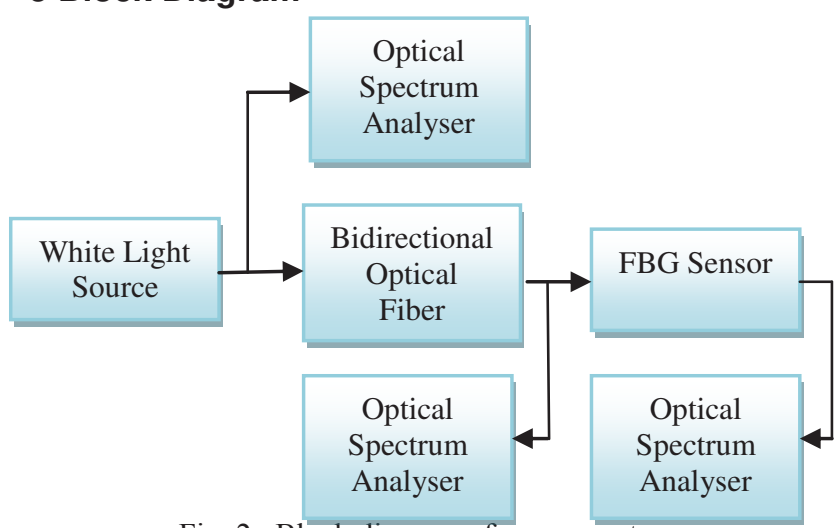

Fig. 2. Block diagram of sensor system

The block diagram of sensor system is as depicted in Fig.2. 
The design consists of white light source, Fiber Bragg grating sensor, Optical delay and Spectrum analyzer.

A white light source is selected because as light source it has broad optical bandwidth and emits visible white light. Optical delay is used to provide precision optical path variation, it controls the delay through the device by varying the distance the light travels between the input and output power. To measure and display the distribution of power of an optical source Optical spectrum analyzer is used. Optical power is displayed on vertical axis while frequency is displayed along horizontal axis.

\subsection{Parameter selection}

The parameter selection is as shown in Table 1.

Table 1. Parameter Selection

\begin{tabular}{|l|l|}
\hline \multicolumn{1}{|c|}{ Name } & \multicolumn{1}{c|}{ Specification } \\
\hline White light source & $\begin{array}{l}\text { Frequency } 193.1 \mathrm{THz} \text { and } \\
\text { Power }-130 \mathrm{dBm}\end{array}$ \\
\hline $\begin{array}{l}\text { Bidirectional Optical Fiber } \\
\text { length }\end{array}$ & $0.002 \mathrm{Km}$ \\
\hline Uniform FBG sensor & at $1550 \mathrm{~nm}$ \\
\hline Optical Null & One \\
\hline Optical Spectral Analyzer & Three \\
\hline
\end{tabular}

The parameters are selected as mentioned in Table 1 . White light source at frequency of $193.1 \mathrm{THz}$ and of power $-130 \mathrm{dBm}$ is used as optical source. Bidirectional Optical fiber is of length $0.002 \mathrm{Km}$ is used.

FBG sensor is used at $1550 \mathrm{~nm}$.Optical null is used for accuracy measurement. Optical spectral analyzers are used for spectrum analysis of input signal, transmitted signal and reflected signal. Optical null is used to improve the accuracy of optical measurement.

A simplified architecture of fiber optic sensor system based on FBG is illustrated in Fig.3.

The simulations are carried out as per selected blocks and design parameters.

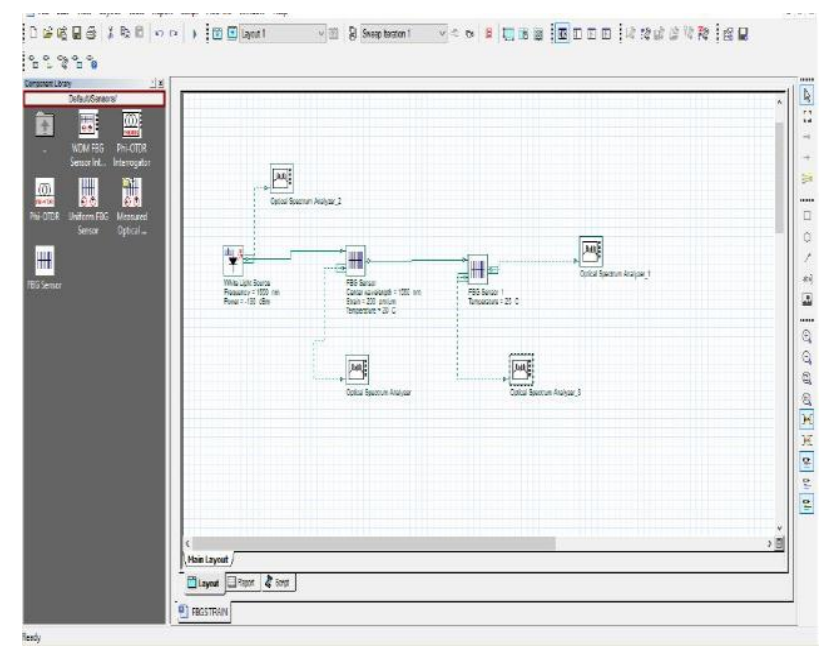

Fig. 3. A simplified architecture of fiber optic sensor

\section{Results and Discussion}

Total six simulations for six different values of strain are carried out to analyse the performance of FBG based sensor and corresponding wavelength shifts using Optisystem software. Power spectrum of input signal is depicted in Fig.4

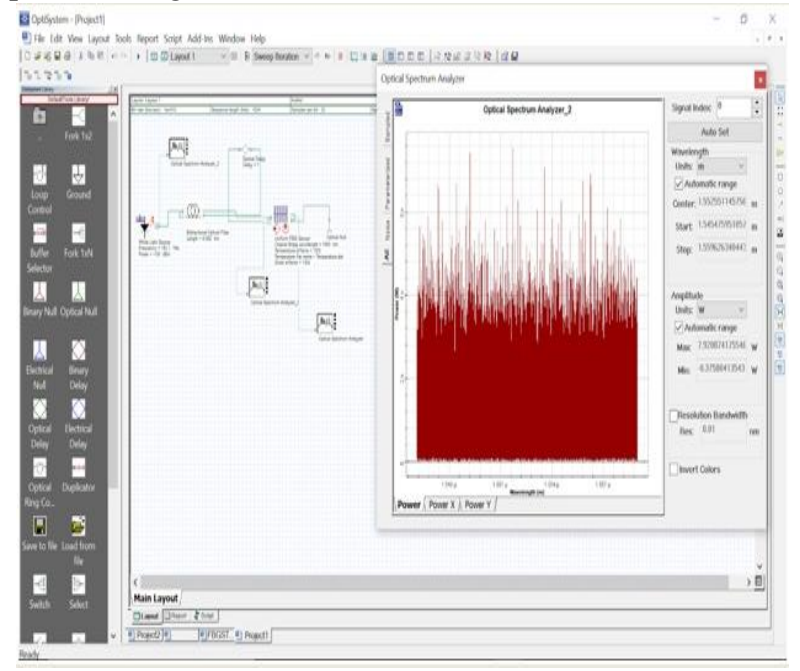

Fig. 4. Power spectrum of Input signal

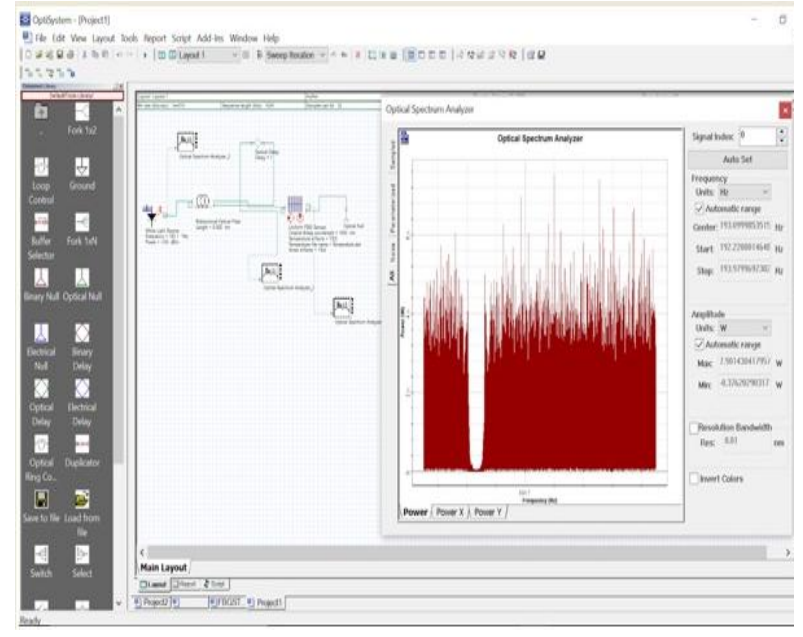

Fig. 5. Power spectrum of transmitted signal

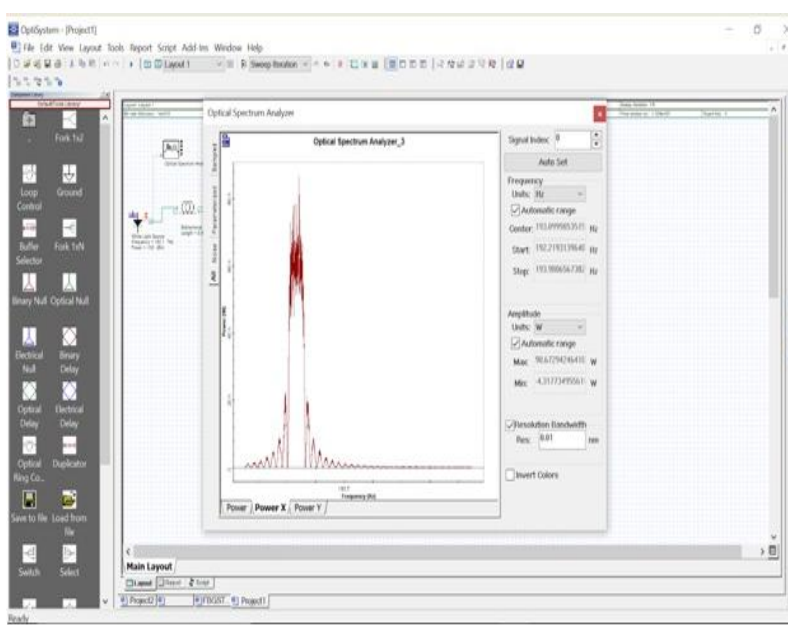

Fig. 6. Power spectrum of reflected signal 
The transmitted signals and reflected signal power spectrum is depicted in Fig. 5 and Fig. 6

Table 2. Wavelength shifting (Theoretical result)

\begin{tabular}{|c|c|c|c|}
\hline $\begin{array}{l}\text { Initial } \\
\text { Wavelength } \\
\lambda \mathrm{B}(\mathrm{nm})\end{array}$ & $\begin{array}{l}\text { Temperature } \\
\text { in }{ }^{0} \mathrm{C}\end{array}$ & $\begin{array}{l}\text { Strain } \\
\text { Experienced } \\
\text { by FBG }\end{array}$ & $\begin{array}{l}\text { Wavelength } \\
\text { Shifted } \Delta \lambda \text { in } \\
(\mu \mathrm{m})\end{array}$ \\
\hline 1550 & 20 & 0.005 & 0.01010 \\
\hline 1550 & 20 & 0.006 & 0.01134 \\
\hline 1550 & 20 & 0.007 & 0.01258 \\
\hline 1550 & 20 & 0.008 & 0.01382 \\
\hline 1550 & 20 & 0.009 & 0.01506 \\
\hline 1550 & 20 & 0.001 & 0.01630 \\
\hline
\end{tabular}

Table 3. Wavelength shifting (simulation result)

\begin{tabular}{|c|c|c|c|}
\hline $\begin{array}{l}\text { Initial } \\
\text { Wavelength } \\
\lambda \mathrm{B}(\mathrm{nm})\end{array}$ & $\begin{array}{l}\text { Temperature } \\
\text { in }{ }^{0} \mathrm{C}\end{array}$ & $\begin{array}{l}\text { Strain } \\
\text { Experienced } \\
\text { by FBG }\end{array}$ & $\begin{array}{l}\text { Wavelength } \\
\text { Shifted } \Delta \lambda \text { in } \\
(\mu \mathrm{m})\end{array}$ \\
\hline 1550 & 20 & 0.005 & 0.0101 \\
\hline 1550 & 20 & 0.006 & 0.01142 \\
\hline 1550 & 20 & 0.007 & 0.01235 \\
\hline 1550 & 20 & 0.008 & 0.0136 \\
\hline 1550 & 20 & 0.009 & 0.0142 \\
\hline 1550 & 20 & 0.001 & 0.01520 \\
\hline
\end{tabular}

The initial wavelength is considered as $1550 \mathrm{~nm}$ and temperature is considered as $20^{\circ} \mathrm{C}$. The wavelength shifting for theoretical result and the wavelength shifted simulation result are illustrated in Table 2 and Table 3. The theoretical and simulated results are closure. The proposed system is simulated at constant temperature.

\section{Conclusion}

Simulation of FBG based sensor system is successfully demonstrated, it has been observed that FBG sensor can be used to measure strain analysis. A Fiber Bragg Grating based sensors can be used for strain analysis. The wavelength of FBG sensors changes with change in the refractive index. Thus any physical parameter can be sensed by using FBG, the simulation tool can provide valuable inputs ahead of essentially engraving the grating in the fiber, in optimizing the design parameters of FBG based sensors. The FBG based sensors has capability to replace existing traditional sensors.

\section{References}

1. O. Andreas, K. Kyriacos, Fiber Bragg Grating. Fundamentals and Applications in Telecommunications and Sensing, Artech House (1999).

2. Yu, F.T.S. Yin, S. Fiber Optic Sensors Marcel Dekker Ltd.: New York, NY, USA(2002).

3. G. Wild S. Hinckley and P. Jansz, A transmit reflectdetection system for fiber Bragg grating hotonicsensors, Proc. SPIE,20 6801(2008).

4. K.S.Khaled, M.Zafrullah; S.M.Bilal,M.A.Merza, Analysis of Gaussian Apodized Fiber Bragg Grating Sensor, Journal of Optical Technology, 79, 7785(2012).

5. Kuse, N. Ozawa, A.; Kobayashi, Y. Static FBG strain sensor with high resolution and large dynamic range by dual-comb spectroscopy. Opt. Express, 21, 11141(2013).

6. Wang, Y., Qiao, X.; Yang, H., Su, D. Li, L. Guo, T. Sensitivity-Improved Strain Sensor over a Large Range of Temperatures Using an Etched and Regenerated Fiber Bragg Grating. Sensors 14, 18575-18582(2014).

7. Catalano, A., Bruno, F.; Pisco, M., Cutolo, A. Cusano, A. An Intrusion Detection System for the Protection of Railway Assets Using Fiber Bragg Grating Sensors. Sensors 14, 18268-18285(2014).

8. Bhowmik, K.; Peng, G.D.; Luo, Y.; Ambikairajah, E.; Lovric, V., Walsh, W.R.; Rajan, G. High Intrinsic Sensitivity Etched Polymer Fiber Bragg Grating Pair for Simultaneous Strain and Temperature Measurements. IEEE Sens. J., 16, 2453-2459(2016).

9. Chen, J. Liu, Q. He Z. High-Resolution Simultaneous Measurement of Strain and Temperature Using pi-Phase-Shifted FBG in Polarization Maintaining Fiber. J. Lightw. Technol. , 35, 4838-4844(2017).

10. Joe, H.E. Yun, H. Jo, S.H. Jun, M.B. Min, B.K. A review on optical fiber sensors for environmental monitoring. Int. J. Precis. Eng. Manuf. Green Technology. 2018, 5, 173-191(2018). 